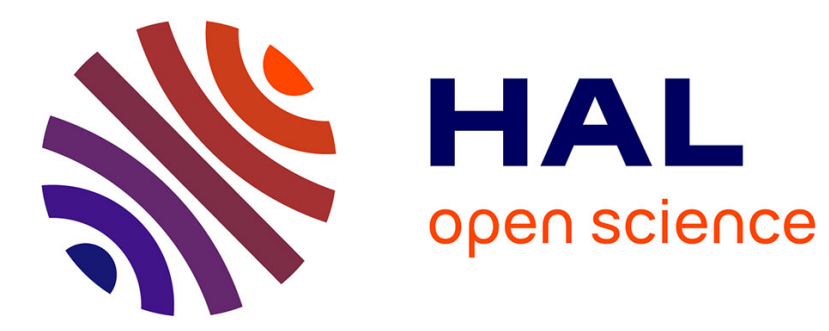

\title{
Learning from evolved next release problem instances
}

Zhilei Ren, He Jiang, Jifeng Xuan, Shuwei Zhang, Zhongxuan Luo

\section{To cite this version:}

Zhilei Ren, He Jiang, Jifeng Xuan, Shuwei Zhang, Zhongxuan Luo. Learning from evolved next release problem instances. GECCO - Genetic and Evolutionary Computation Conference, 2014, ACM SIGEVO, Jul 2014, Vancouver, BC, Canada. pp.189 - 190, 10.1145/2598394.2598427 . hal-01087436

\section{HAL Id: hal-01087436 https://hal.inria.fr/hal-01087436}

Submitted on 26 Nov 2014

HAL is a multi-disciplinary open access archive for the deposit and dissemination of scientific research documents, whether they are published or not. The documents may come from teaching and research institutions in France or abroad, or from public or private research centers.
L'archive ouverte pluridisciplinaire $\mathbf{H A L}$, est destinée au dépôt et à la diffusion de documents scientifiques de niveau recherche, publiés ou non, émanant des établissements d'enseignement et de recherche français ou étrangers, des laboratoires publics ou privés. 


\section{Learning from Evolved Next Release Problem Instances}

\author{
Zhilei Ren He Jiang \\ Dalian University of Technology \\ Dalian 116623, China \\ \{zren, jianghe\}@dlut.edu.cn
}

\author{
Jifeng Xuan \\ INRIA Lille - Nord Europe \\ Lille 59650, France \\ jifeng.xuan@inria.fr
}

\author{
Shuwei Zhang Zhongxuan Luo \\ Dalian University of Technology \\ Dalian 116623, China \\ zhangshuwei@mail.dlut.edu.cn \\ zxluo@dlut.edu.cn
}

\begin{abstract}
Taking the Next Release Problem (NRP) as a case study, we intend to analyze the relationship between heuristics and the software engineering problem instances. We adopt an evolutionary algorithm to evolve NRP instances that are either hard or easy for the target heuristic (GRASP in this study), to investigate where a heuristic works well and where it does not, when facing a software engineering problem. Thereafter, we use a feature-based approach to predict the hardness of the evolved instances, with respect to the target heuristic. Experimental results reveal that, the proposed algorithm is able to evolve NRP instances with different hardness. Furthermore, the problem-specific features enables the prediction of the target heuristic's performance.
\end{abstract}

\section{Categories and Subject Descriptors}

D.2.1 [Software Engineering]: Requirements/ Specifications-Methodologies

\section{Keywords}

Genetic algorithm, Next release problem, Problem hardness

\section{INTRODUCTION}

In this study, we take the Next Release Problem (NRP) as a case study, to analyze the relationship between heuristic algorithms and the software engineering problem instance hardness. We are interested in investigating both the strength and the weakness of a given heuristic, when facing a software engineering problem. To achieve this, we use a genetic algorithm to evolve a set of NRP instances that are hard and easy to solve, respectively, with respect to the target heuristic. Then, we extract a set of features from the evolved instances, to capture the distribution information of the instance specific variables. With these proposed features, we are able to predict the target heuristic's performance.

\section{THE NEXT RELEASE PROBLEM}

In a software project, let $R$ be the set of candidate requirements with $|R|=m$. Each requirement $r_{j} \in R(1 \leq$ $j \leq m$ ) corresponds to a nonnegative cost $c_{j} \in C$. A directed acyclic graph $G=(R, E)$ indicates the dependencies between these requirements, with $R$ denoting the vertices and $E$ specifying the arcs. In the dependency graph $G$, an $\operatorname{arc}\left(r^{\prime}, r\right) \in E$ means that the requirement $r$ relies on $r^{\prime}$. We call the requirement $r$ the child requirement of $r^{\prime}$. parents $(r)$ is defined as parents $(r)=\left\{r^{\prime} \in R \mid\left(r^{\prime}, r^{\prime \prime}\right) \in\right.$
$\left.E, r^{\prime \prime} \in \operatorname{parents}(r)\right\}$. All requirements of parents $(r)$ have to be realized to ensure that $r$ is implemented.

Let $S$ be the customer set with $|S|=n$. Each customer $s_{i} \in S$, requests a subset of requirements $R_{i} \subseteq R$. Let $w_{i} \in W$ be the weight for the customer $s_{i}$. Let parents $\left(R_{i}\right)$ be $\bigcup_{r \in R_{i}}$ parents $(r)$. Given a customer $s_{i}$, let the set of requirements requested by $s_{i}$ be $\hat{R}_{i}=R_{i} \bigcup$ parents $\left(R_{i}\right)$. Hence, a customer $s_{i}$ can be satisfied if and only if all the requirements in $R_{i}$ are realized during the next release. Let the cost of satisfying the customer $s_{i}$ be $\operatorname{cost}\left(\hat{R}_{i}\right)=\sum_{r_{j} \in \hat{R}_{i}} c_{j}$. The NRP is formulated as follows. Given a set of customers $\left\{y_{1}, \cdots, y_{n}\right\}$ and a set of requirements $\left\{x_{1}, \cdots, x_{m}\right\}$, as well as the corresponding weights $\left\{w_{1}, \cdots, w_{n}\right\}$ and cost $\left\{c_{1}, \cdots, c_{m}\right\}$, the NRP aims to select a subset of customers, to maximize the corresponding weights. Without loss of generality, in this study we assume that the customers are sorted in descending order with respect to their weight-to-cost ra$\operatorname{tios}\left(\frac{w_{1}}{\operatorname{cost}\left(\hat{R_{1}}\right)}>\frac{w_{2}}{\operatorname{cost}\left(\hat{R_{2}}\right)}>\cdots>\frac{w_{m}}{\operatorname{cost}\left(\hat{R_{m}}\right)}\right)$. The objective of the NRP is to maximize the following formulations:

$$
\sum_{i=1}^{m} w_{i} y_{i}
$$

subject to

$$
\begin{gathered}
\sum_{i=1}^{n} c_{i} x_{i} \leq B, \\
x_{i} \geq x_{j}, \forall\left(r_{i}, r_{j}\right) \in E, \\
x_{i} \geq y_{j}, \forall i, j \text { where } r_{i} \text { is required by customer } j . \\
x_{i}, y_{j} \in\{0,1\}, 1 \leq i \leq n, 1 \leq j \leq m .
\end{gathered}
$$

\section{EVOLUTIONARY ALGORITHM TO GEN- ERATE NRP INSTANCES}

A genetic algorithm is employed to evolve NRP instances, which are hard or easy to solve, respectively. The algorithm maintains a population of 20 individuals. Each individual is represented by an NRP instance. More specifically, the variables of each individual involve the values of the weights and the costs. The other instance specific parameters, such as the number of customers, the budget bound, and the dependency relationships are inherited from the input seed instance. For the genetic operators, we employ the single point crossover, and the uniform mutation with the mutation rate 0.05. For the selection method, we adopt the truncation selection. The fitness value of a given instance is assigned 
by the mean error (compared against the optimal solution), which is achieved by 30 independent runs of GRASP [2].

\section{CAPTURING THE NRP CHARACTER- ISTICS USING FEATURES}

We consider two classes of features that may influence the instance hardness. The first set of features include the correlation between each customer's weight and the corresponding costs, the standard deviation of the customers' weights, the standard deviation of the costs, the standard deviation of the weight-to-cost ratio, the coefficient of variation of all the customers' weights, the coefficient of variation of the costs, and the coefficient of variation of the weight-to-cost ratio. The second set of features are extracted from the relaxed version of the NRP. The motivation of these features are inspired by the core concept from the knapsack problem literatures [4]. As mentioned, in this study, we assume the customers are sorted according to the weight-to-cost ratio. Furthermore, due to the intrinsic difficulty of the NRP, we consider the relaxed version of the NRP in [2], i.e., we replace constraint (5) with the following constraint:

$$
x_{i} \in[0,1], y_{j} \in[0,1], 1 \leq i \leq n, 1 \leq j \leq m .
$$

Given the optimal solution $\left\{\hat{y}_{1}, \cdots, \hat{y}_{m}\right\}$ to the relaxed NRP, we define $\hat{j}_{1}, \hat{j}_{2}, \hat{j}_{\text {min }}, \hat{j}_{\text {max }}$ as follows: $\hat{j}_{1}=\min \{1 \leq$ $\left.j \leq m \mid \hat{y}_{j}=0\right\}, \hat{j}_{2}=\max \left\{1 \leq j \leq m \mid \hat{y}_{j}>0\right\}, \hat{j}_{\min }=$ $\min \left\{\hat{j}_{1}, \hat{j}_{2}\right\}, \hat{j}_{\max }=\max \left\{\hat{j}_{1}, \hat{j}_{2}\right\}$. With the auxiliary variables $\hat{j}_{\text {min }}$ and $\hat{j}_{\text {max }}$, the customer set could be partitioned into three subsets (corresponding to customers with index ranging from $\left[1, \hat{j}_{\text {min }}\right),\left[\hat{j}_{\text {min }}, \hat{j}_{\max }\right]$, and $\left.\left(\hat{j}_{\max }, m\right]\right)$. The rescaled sum of weights $\left(\sigma_{1}-\sigma_{3}\right)$ and the rescaled sum of costs $\left(\delta_{1}-\delta_{3}\right)$ are defined as follows: $\sigma_{1}=\frac{\sum_{1 \leq i<\hat{j}_{\text {min }} w_{i}} w_{i}}{\sum_{1 \leq i \leq m} w_{i}}$,

$$
\begin{aligned}
& \sigma_{2}=\frac{\sum_{\text {min }} \leq i \leq \hat{j}_{\max } w_{i}}{\sum_{1 \leq i \leq m} w_{i}}, \sigma_{3}=\frac{\sum_{\text {max }} \sum_{i \leq m} w_{i}}{\sum_{1 \leq i \leq m} w_{i}}, \delta_{1}=\frac{\sum_{1 \leq i<\hat{j}_{\min }} \operatorname{cost}\left(\hat{R}_{i}\right)}{\sum_{1 \leq i \leq n} c_{i}} \text {, } \\
& \delta_{2}=\frac{\sum_{\hat{j}_{\text {min }} \leq i \leq \hat{j}_{\text {max }}} \operatorname{cost}\left(\hat{R}_{i}\right)}{\sum_{1 \leq i \leq n} c_{i}}, \delta_{3}=\frac{\sum_{\hat{j}_{\text {max }}<i \leq m} \operatorname{cost}\left(\hat{R}_{i}\right)}{\sum_{1 \leq i \leq n} c_{i}} \text {. Also, the }
\end{aligned}
$$

upper bound obtained from the optimal solution to the relaxed NRP instance is used as a feature.

\section{EXPERIMENTAL RESULTS}

To predict the target heuristic's performance, we adopt the $\mathrm{R}$ port of random forest model randomForest [3]. We employ the open source package SCIP [1] as the solver to obtain the optimal solutions. For the seed instances, we use the benchmark instances from [2]. To evolve hard NRP instances, we execute the algorithm over each seed instance for 10 independent runs. The easy NRP instances are generated similarly. Hence, we obtain 150 hard NRP instances and 150 easy NRP instances, respectively. Furthermore, for both the hard and the easy instances, we randomly select $50 \%$ of the instances as the training instances, and treat the rest of the instances as the test instances.

We first investigate the distribution of the mean error of the target heuristic over the two sets of evolved instances. We notice that the median difficulty of the hard instance set is significantly higher than the easy instance set. Besides, we find that the mean error achieved by the hard instance set has a larger variation (ranging from around 0.1 to above $0.5)$, while the mean error for the easy instance set has a

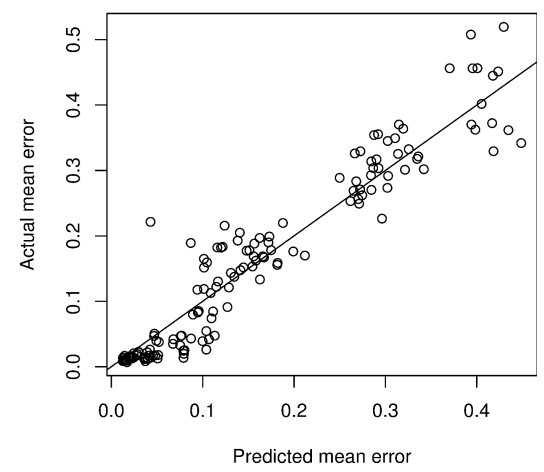

Figure 1: Comparison between the predicted performance and the actual performance

relative small variation. Then, we investigate the possibility of predicting the solution quality. To achieve this, we use the random forest regression model. Fig. 1 presents the results of the regression model. In the figure, the $\mathrm{x}$-axis and the $\mathrm{y}$-axis indicate the predicted mean error and the mean error achieved by the target heuristic. Each point indicates the results over one or more instances. From the figure, we observe that the regression model is able to achieve good prediction quality. Most points in the figure lie around the reference line $(y=x)$. Furthermore, the Root Mean Squared Error obtained by the regression model is 0.0409 .

\section{CONCLUSIONS AND FUTURE WORK}

In this paper, we focus on evolving hard and easy requirements engineering problem instances. Given the target heuristic (GRASP), we develop an evolutionary algorithm to generate hard and easy NRP instances, respectively. We also investigate the possibility of predicting the performance of the target heuristic over the evolved NRP instances. For the future work, we are interested in leveraging the problemspecific features to guide the algorithm design process, using approaches such as hyper-heuristics [5].

\section{REFERENCES}

[1] T. Achterberg. SCIP: solving constraint integer programs. Mathematical Programming Computation, 1(1):1-41, 2009.

[2] A. J. Bagnall, V. J. Rayward-Smith, and I. M. Whittley. The next release problem. Information and Software Technology, 43(14):883-890, 2001.

[3] A. Liaw and M. Wiener. Classification and regression by randomforest. $R$ News, 2(3):18-22, 2002.

[4] D. Pisinger. Core problems in knapsack algorithms. Operations Research, 47(4):570-575, 1999.

[5] Z. Ren, H. Jiang, J. Xuan, and Z. Luo. Hyper-heuristics with low level parameter adaptation. Evolutionary computation, 20(2):189-227, 2012. 